\title{
MODELLING OF BEAM-BEAM EFFECTS IN MULTISCALES
}

\author{
Antonina N. Fedorova, Michael G. Zeitlin \\ IPME, RAS, V.O. Bolshoj pr., 61, 199178, St. Petersburg, Russia *†
}

\begin{abstract}
We present the applications of nonlinear local harmonic analysis methods to the modelling of beam-beam interaction. Our approach is based on methods provided the possibility to work with dynamical beam localization in phase space. The consideration of Fokker-Planck or VlasovMaxwell models is based on a number of anzatzes, which reduce initial problems to a number of dynamical systems (with constraints) and on variational-wavelet approach to polynomial/rational approximations for reduced nonlinear dynamics. We calculate contribution to full dynamics (partition function) from all underlying subscales via nonlinear eigenmodes decomposition.
\end{abstract}

\section{INTRODUCTION}

In this paper we consider the applications of numerical-analytical technique based on the methods of local nonlinear harmonic analysis or wavelet analysis to nonlinear models of beam-beam interactions which can be characterized by collective type behaviour. We consider different but related models [1] of the beam-beam interaction from the point of view of construction of reasonable numerical-analytical approaches. It is very important because some beam-beam effects limit luminosity and stability of collider. Such approaches may be useful in all models in which it is possible and reasonable to reduce all complicated problems related with statistical distributions to the problems described by systems of nonlinear ordinary/partial differential equations with or without some (functional) constraints. Wavelet analysis is a set of mathematical methods, which gives us the possibility to work with well-localized bases in functional spaces and gives the maximum sparse forms for the general type of operators (differential, integral, pseudodifferential) in such bases. Our approach is based on the variational-wavelet approach from [2]-[13], which allows us to consider polynomial and rational type of nonlinearities. The constructed solution has the multiscale/multiresolution decomposition via nonlinear high-localized eigenmodes. In this way we give contribution to our full solution from each scale of resolution or each time/(phase) space scale or from each nonlinear eigenmode. Fig.1 demonstrates such decomposition for the finite kick term. The same is correct for the contribution to power spectral density (energy spectrum): we can take into account contribution from each level/scale of resolution. In all models below numerical modelling

\footnotetext{
*e-mail: zeitlin@math.ipme.ru

${ }^{\dagger}$ http://www.ipme.ru/zeitlin.html; http://www.ipme.nw.ru/zeitlin.html
}

demonstrates the appearance of (coherent) high-localized structures and (stable) pattern formation. Starting in part 2 from beam-beam interaction models [1] we consider in part 3 the approach based on variational-wavelet formulation. We give explicit representation for all dynamical variables in the base of compactly supported wavelets or nonlinear eigenmodes. Our solutions are parametrized by solutions of a number of reduced algebraical problems one from which is nonlinear with the same degree of nonlinearity as initial models and the rest are the linear problems which correspond to concrete details of wavelet calculations. In part 4 we consider numerical modelling based on our analytical approach.

\section{BEAM-BEAM MODELLING}

In A. Chao e.a. model [1] for the simulation of beambeam interaction the initial collective description by some sort of Vlasov-Maxwell equation for distribution function $f(s, x, p)$

$$
\frac{\partial f}{\partial s}+p \frac{\partial f}{\partial x}-(k(s) x-F(x, s, f)) \frac{\partial f}{\partial p}=0
$$

is reduced to Fokker-Planck (FP) equation on the first stage

$$
\begin{aligned}
& \frac{\partial f_{k}}{\partial s}-\Gamma_{k} f_{k}+p \frac{\partial f_{k}}{\partial x}- \\
& \left(F_{k}(s, x, f)-G_{k}(s, p)\right) \frac{\partial f_{k}}{\partial p}=D \frac{\partial^{2} f_{k}}{\partial p^{2}}
\end{aligned}
$$

and to some nontrivial dynamical system with complex behaviour

$$
\begin{aligned}
& \frac{\mathrm{d}^{2} \sigma_{k}}{\mathrm{~d} s^{2}}+\Gamma_{k} \frac{\mathrm{d} \sigma_{k}}{\mathrm{~d} s}+F_{k} \sigma_{k}=\frac{1}{\beta_{k}^{2} a_{k}^{2} \sigma_{k}^{3}} \\
& \frac{\mathrm{d} a_{n}}{\mathrm{~d} s}=\Gamma_{k} a_{k}\left(1-a_{k}^{2} \sigma_{k}^{2}\right)
\end{aligned}
$$

on the second stage. Its solution gives the parameters of enveloping gaussian anzatz for solution of FP equation. Related model of R. Davidson e.a.[1] is based on VlasovMaxwell equations:

$$
\begin{aligned}
& \frac{\partial f_{k}}{\partial s}+\nu_{k} p \frac{\partial f_{k}}{\partial x}-\frac{\partial H_{k}}{\partial x} \frac{\partial f_{k}}{\partial p}=0 \\
& H_{k}=\frac{\nu_{k}}{2}\left(p^{2}+x^{2}\right)+\lambda_{k} \delta_{p}(s) V_{k}(x, s) \\
& \frac{\partial^{2} V_{k}}{\partial x^{2}}=4 \pi \int \mathrm{d} p f_{3-k}(x, p, s)
\end{aligned}
$$




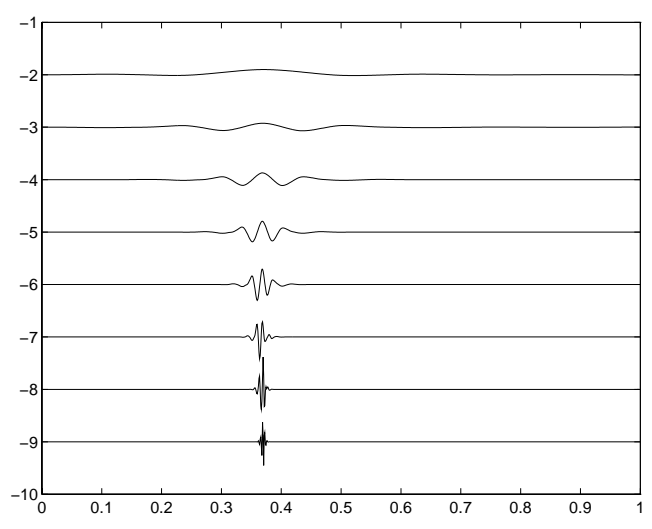

Figure 1: Finite kick decomposition.

\section{VARIATIONAL MULTISCALE REPRESENTATION}

We obtain our multiscale/multiresolution representations (formulae (9) below) for solutions of equations (1),(2),(4) via variational-wavelet approach for the following formal systems of equations (with the corresponding obvious constraints on distribution function), which is the general form of these equations.

Let $\mathrm{L}$ be an arbitrary (non) linear differential/integral operator with matrix dimension $d$, which acts on some set of functions $\quad \Psi \equiv \Psi(s, x, p)=\left(\Psi^{1}(s, x, p), \ldots\right.$, $\left.\Psi^{d}(s, x, p)\right), s, x, p \in \Omega \subset \mathbf{R}^{3}$ from $L^{2}(\Omega):$

$$
L \Psi \equiv L(Q, s, x, p) \Psi(s, x, p)=0,
$$

where

$$
\begin{aligned}
& Q \equiv Q_{d_{1}, d_{2}, d_{3}}(s, x, p, \partial / \partial s, \partial / \partial x, \partial / \partial p)= \\
& \sum_{i_{1}, i_{2}, i_{3}=1}^{d_{1}, d_{2}, d_{3}} a_{i_{1} i_{2} i_{3}}(s, x, p)\left(\frac{\partial}{\partial s}\right)^{i_{1}}\left(\frac{\partial}{\partial x}\right)^{i_{2}}\left(\frac{\partial}{\partial p}\right)^{i_{3}}
\end{aligned}
$$

Let us consider now the $\mathrm{N}$ mode approximation for solution as the following ansatz (in the same way we may consider different ansatzes):

$$
\Psi^{N}(s, x, p)=\sum_{r, s, k=1}^{N} a_{r s k} A_{r}(s) \otimes B_{s}(x) \otimes C_{k}(p)
$$

We shall determine the coefficients of expansion from the following conditions (different related variational approaches are considered in [2]-[13]):

$$
\ell_{k \ell m}^{N} \equiv \int\left(L \Psi^{N}\right) A_{k}(s) B_{\ell}(x) C_{m}(p) \mathrm{d} s \mathrm{~d} x \mathrm{~d} p=0
$$

So, we have exactly $d N^{3}$ algebraical equations for $d N^{3}$ unknowns $a_{r s k}$. Such variational approach reduces the initial problem to the problem of solution of functional equations at the first stage and some algebraical problems at the second stage. We consider the multiresolution expansion as the second main part of our construction. The solution is parametrized by solutions of two set of reduced algebraical problems, one is linear or nonlinear (depends on the structure of operator $\mathrm{L}$ ) and the rest are some linear problems related to computation of coefficients of algebraic equations (8). These coefficients can be found by some wavelet methods by using compactly supported wavelet basis functions for expansions (7). We may consider also different types of wavelets including general wavelet packets [2]-[13]. The constructed solution has the following multiscale/multiresolution decomposition via nonlinear high-localized eigenmodes

$$
\begin{aligned}
& f(s, x, p)=\sum_{(i, j, k) \in Z^{3}} a_{i j k} A^{i}(s) B^{j}(x) C^{k}(p), \\
& A^{i}(s)=A_{N}^{i, s l o w}(s)+\sum_{r \geq N} A_{r}^{i}\left(\omega_{r}^{1} s\right), \omega_{r}^{1} \sim 2^{r} \\
& B^{j}(x)=B_{M}^{j, s l o w}(x)+\sum_{l \geq M} B_{l}^{j}\left(\omega_{l}^{2} x\right), \omega_{l}^{2} \sim 2^{l} \\
& C^{k}(p)=C_{K}^{k, \text { slow }}(p)+\sum_{m \geq K} C_{m}^{k}\left(\omega_{m}^{3} p\right), \omega_{m}^{3} \sim 2^{m}
\end{aligned}
$$

which corresponds to the full multiresolution expansion in all underlying time/space scales. Formula (9) gives us expansion into the slow part $f_{N, M, K}^{s l o w}$ and fast oscillating parts for arbitrary N, M, K. So, we may move from coarse scales of resolution to the finest one to obtain more detailed information about our dynamical process. The first terms in the RHS of formulae (9) correspond on the global level of function space decomposition to resolution space and the second ones to detail space. Particular one-dimensional case of formulae (9) determines the solution of equations (3) (more exactly corresponding constructions are considered in other papers presented during this Conference). But, it should be noted that in this one-dimensional case we have really nontrivial nonlinear dynamics only for functional parameters of enveloping gaussians, which give the solution only for linearization of equations (1),(2),(4). As we demonstrated, our representation (9) provides the solution as in linear as in nonlinear cases without any perturbation technique but on the level of expansions in (functional) space of solutions. The using of wavelet basis with highlocalized properties provides good convergence properties of constructed solution (9). Because affine group of translation and dilations is inside the approach, this method resembles the action of a microscope. We have contribution to final result from each scale of resolution from the whole infinite scale of spaces or from each underlying scale: the closed subspace $V_{j}(j \in \mathbf{Z})$ corresponds to level $\mathbf{j}$ of resolution, or to scale j. Our full multiresolution/multiscale decomposition of functional space $L^{2}\left(\mathbf{R}^{n}\right)$ of solutions of initial problems, which is a sequence of increasing closed subspaces $V_{j}: \ldots V_{-2} \subset V_{-1} \subset V_{0} \subset V_{1} \subset V_{2} \subset \ldots$, provides us with formulae (9). This functional space decomposition corresponds to (exact) nonlinear eigenmode decomposition. It should be noted that such representations give the best possible localization properties in the 
corresponding (phase)space/time coordinates. In contrast with different approaches, formulae (9) does not use perturbation technique or linearization procedures and represents dynamics via generalized nonlinear localized eigenmodes expansion. So, by using wavelet bases with their good (phase)space/time localization properties we can construct high-localized coherent structures in spatially-extended stochastic systems with collective behaviour.

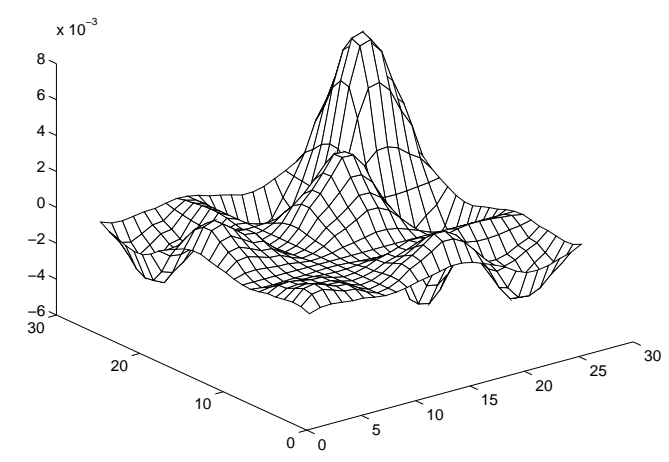

Figure 2: Eigenmode of level 1.

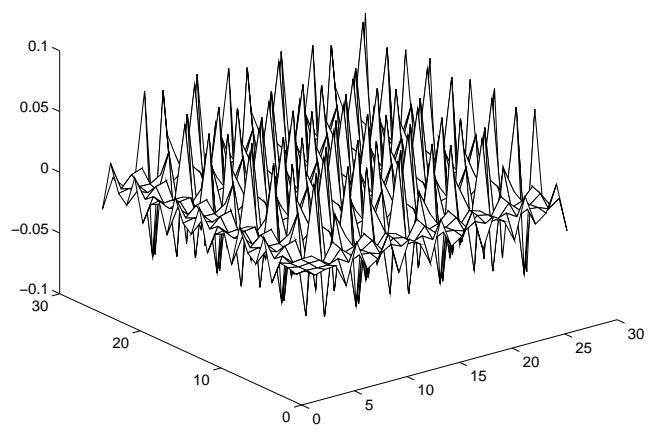

Figure 3: Eigenmode of level 4.

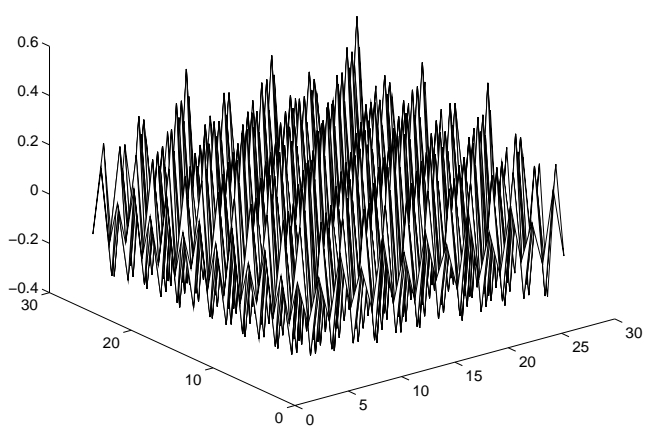

Figure 4: Six-eigenmodes representation.

\section{MODELLING}

Multiresolution/multiscale representations for solutions of equations from part 2 in the high-localized bases/eigenmodes are demonstrated on Fig. 2-Fig. 4. On Fig. 2, Fig. 3 we present contribution to the full expansion (9) from level 1 and level 4 of full decomposition. Fig. 4 show the representation for full solution, constructed from the first 6 eigenmodes (6 levels in formula (9)).

\section{ACKNOWLEDGMENTS}

We would like to thank The U.S. Civilian Research \& Development Foundation (CRDF) for support (Grants TGP-454, 455), which gave us the possibility to present our nine papers during PAC2001 Conference in Chicago and Ms. Camille de Walder from CRDF for her help and encouragement.

\section{REFERENCES}

[1] A. Chao, e.a., Los Alamos preprint, physics/0010055. R.C. Davidson, e.a., Los Alamos preprint, physics/0104086.

[2] A.N. Fedorova and M.G. Zeitlin, Math. and Comp. in Simulation, 46, 527, 1998.

[3] A.N. Fedorova and M.G. Zeitlin, New Applications of Nonlinear and Chaotic Dynamics in Mechanics, 31, $101 \mathrm{Klu}-$ wer, 1998.

[4] A.N. Fedorova and M.G. Zeitlin, CP405, 87, American Institute of Physics, 1997. Los Alamos preprint, physics/9710035.

[5] A.N. Fedorova, M.G. Zeitlin and Z. Parsa, Proc. PAC97 2, 1502, 1505, 1508, APS/IEEE, 1998.

[6] A.N. Fedorova, M.G. Zeitlin and Z. Parsa, Proc. EPAC98, 930, 933, Institute of Physics, 1998.

[7] A.N. Fedorova, M.G. Zeitlin and Z. Parsa, CP468, 48, American Institute of Physics, 1999. Los Alamos preprint, physics/990262.

[8] A.N. Fedorova, M.G. Zeitlin and Z. Parsa, CP468, 69, American Institute of Physics, 1999. Los Alamos preprint, physics/990263.

[9] A.N. Fedorova and M.G. Zeitlin, Proc. PAC99, 1614, 1617, 1620, 2900, 2903, 2906, 2909, 2912, APS/IEEE, New York, 1999.

Los Alamos preprints: physics/9904039, 9904040, 9904041, 9904042, 9904043, 9904045, 9904046, 9904047.

[10] A.N. Fedorova and M.G. Zeitlin, The Physics of High Brightness Beams, 235, World Scientific, 2000. Los Alamos preprint: physics/0003095.

[11] A.N. Fedorova and M.G. Zeitlin, Proc. EPAC00, 415, 872, 1101, 1190, 1339, 2325,Austrian Acad.Sci.,2000.

Los Alamos preprints: physics/0008045, 0008046, 0008047, 0008048, 0008049, 0008050.

[12] A.N. Fedorova, M.G. Zeitlin, Proc. 20 International Linac Conf., 300, 303, SLAC, Stanford, 2000. Los Alamos preprints: physics/0008043, 0008200.

[13] A.N. Fedorova, M.G. Zeitlin, Los Alamos preprints: physics/0101006, 0101007 and World Scientific, in press. 